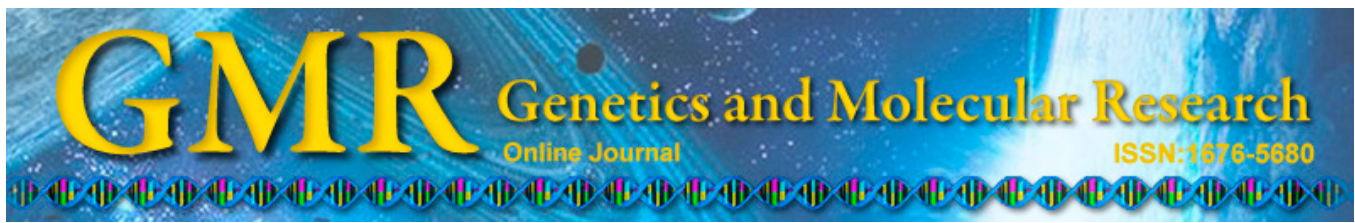

\title{
Bioinformatic analysis of the effect of type II diabetes on skin wound healing
}

\author{
K. Ge ${ }^{1 *}$,J.J. Wu ${ }^{1 *}$, L. Qian ${ }^{1}$, M.J. Wu ${ }^{2}$, F.L. Wang ${ }^{1}$, B. Xu ${ }^{1}$ and T. Xie ${ }^{2}$ \\ ${ }^{1}$ Department of Emergency, Shanghai 9th People's Hospital, \\ Shanghai Jiaotong University School of Medicine, Shanghai, China \\ ${ }^{2}$ Department of Wound Healing, Shanghai 9th People's Hospital, \\ Shanghai Jiaotong University School of Medicine, Shanghai, China \\ *These authors contributed equally to this study. \\ Corresponding author: B. Xu \\ E-mail: bingxubx@163.com
}

Genet. Mol. Res. 14 (2): 4802-4811 (2015)

Received June 17, 2014

Accepted November 18, 2014

Published May 11, 2015

DOI http://dx.doi.org/10.4238/2015.May.11.12

\begin{abstract}
We examined the relationship between type 2 diabetes and skin wound healing. GSE38396 was downloaded from the Gene Expression Omnibus database and preprocessed using the RMA function of the Affy package. Differentially expressed genes (DEGs) were identified using the limma package, then DAVID was applied to perform Gene Ontology functional annotation and Kyoto Encyclopedia of Genes and Genomes pathway enrichment analysis. MicroRNAs and their target genes were screened from the miRecords database and subjected to functional analysis. Finally, the STRING online database was applied to identify the protein-protein interaction relationships, and a combined score $>0.5$ was considered to indicate an interaction. A total of 421 DEGs (208 upregulated and 213 downregulated genes) were identified in the skin lymphatic endothelial cells of patients with type II diabetes. Twenty-four microRNAs and 34 target genes were screened, including those involved in cell migration, regulation of cell proliferation, cell death, and cell adhesion regulation, among others. Protein-
\end{abstract}


protein interaction network clustering analysis identified a module composed of 25 genes, and INTERPRO protein domain enrichment analysis showed that the protein domain of the clustering module mainly contained the insulin-like growth factor binding proteins IGFBP3 and CYR61. IGFBP3 and CYR61 may play important roles in skin wound healing in diabetes patients. This information may be useful for developing methods to treat skin refractory wounds in type II diabetes.

Key words: Cysteine-rich angiogenic inducer 61; Wound healing; Insulin-like growth factor-binding protein 3; Type II diabetes

\section{INTRODUCTION}

With the development of world society and economics, changes in diet, a more intense pace of life, and many other factors, the global incidence and prevalence of diabetes is rapidly increasing. Diabetes is ranked as the third-most common chronic disease threating human health after cancer and cardiovascular disease (Sakai and Tagami, 2010; Buerger et al., 2012). Diabetic skin is vulnerable to damage; once injured, it is often recurrent, even causing intractable refractory ulcers (Spravchikov et al., 2001). Therefore, improved treatments should be developed for the clinical treatment of diabetic ulcers.

Because of recent developments in molecular biology techniques as well as other disciplines, studies examining the mechanisms of diabetic wound healing have become increasingly in-depth, and the signaling pathway, angiogenesis, neuropeptides, advanced glycation end products, apoptosis, and other aspects of matrix metalloproteinases have been intensively investigated (Brem and Tomic-Canic, 2007; Liu and Velazquez, 2008). Transforming growth factor $2 \beta 1$ can inhibit the growth of epithelial cells and accelerate angiogenesis. This growth factor is released in early wound healing; however, its expression decreases in diabetic ulcer regions (Goto et al., 1993). The Ras signaling pathway and $\mathrm{Wnt} / \beta 2$ catenin signaling pathway have been confirmed to play important roles in the mechanisms of diabetic wound healing or delayed healing (Wang et al., 2010; Santarpia et al., 2012). Vascular endothelial growth factor, angiogenic factors 21, basic fibroblast growth factor, and other cytokines are closely related to wound healing (Rapraeger et al., 1991; Chan et al., 2008; Lu and Bergers, 2013). Diabetes may reduce levels of cell endothelial growth factors, further decreasing vasodilation capacity and even leading to microangiopathy (Brownlee, 2001). These results revealed the mechanism of skin wound healing in type II diabetes at the genetic level; however, this mechanism at the microRNA (miRNA) and protein levels are unknown. An understanding of these processes may provide a comprehensive view for the mechanism of wound healing in type II diabetes. To examine the mechanism of skin wound healing in type II diabetes patients, we used a microarray to analyze the expression profile of GSE38396. First, the limma package of $R$ language was used to identify differentially expressed genes (DEGs) for experimental samples and control samples. In addition, the Database for Annotation, Visualization, and Integrated Discovery (DAVID) was applied to perform Gene Ontology (GO) function annotation and Kyoto Encyclopedia of Genes and Genomes (KEGG) pathway enrichment analysis and for the DEGs. Finally, Cytoscape was used to construct the protein-protein interaction network and perform module analysis to identify key genes. 


\section{MATERIAL AND METHODS}

\section{Data resource}

The GSE38396 (Haemmerle et al., 2013), an RNA expressional profile, was downloaded from the Gene Expression Omnibus database, which included 4 experimental samples from skin lymphatic endothelial cells of patients with type II diabetes and 4 control samples from skin lymphatic endothelial cells of patients with non-type II diabetes. The platform of GSE38396 was Affymetrix Human Genome U133 Plus 2.0 Array [GPL570 (HG-U133_ Plus_2)] (Affymetrix, Santa Clara, CA, USA).

\section{Data preprocessing}

The CEL original format of GSE38396 was downloaded from the database, and the RNA data were preprocessed using the Robust Multiarray Average algorithm in the Affy package (Irizarry et al., 2003) to obtain the expression matrix. If different probes were mapped to the same gene, the mean value of the probes was used as the final expression value of this gene.

\section{Identification of DEGs}

The limma package of $\mathrm{R}$ language (Sanges et al., 2007) was applied to screen the DEGs based on the Student $t$-test, and genes with $\mathrm{P}$ value $<0.05$ and $|\operatorname{logFC}|>0.5$ were selected as DEGs.

\section{Gene function annotation}

DAVID (Huang et al., 2008a) was applied to perform GO functional annotation and KEGG pathway enrichment analysis for DEGs, and then significantly enriched GO terms (P value $\leq 0.05$ ) and KEGG pathways (P value $\leq 0.05)$ were identified to explore the effect of type II diabetes on skin wound healing.

\section{Regulatory relationship between miRNA and DEGs}

Gene signatures were extracted from the miRecords database (Xiao et al., 2009) as the miRNAs of the target genes, followed by functional analysis.

\section{Construction and analysis of the protein-protein interaction network}

The Search Tool for the Retrieval of Interacting Genes database (functional protein association networks) (Franceschini et al., 2013) was applied to determine protein-protein interaction relationships, and a combined score of $>0.5$ was selected as the threshold indicating protein interaction relationships. Next, the Cytoscape software (Shannon et al., 2003) was used to construct the protein-protein interaction network. 


\section{Network module analysis}

The module with P value $<1.0 \mathrm{E}-5$ was selected as a functional module using the ClusterONE plug of Cytoscape (Shannon et al., 2003) with default parameters.

\section{RESULTS}

\section{Expression matrix normalization}

The value of the cassette matrix before and after normalization is shown in Figure 1 and the normalized median line was nearly straight, indicating that normalization was successful. After treatment, 19,944 genes remained from the original 54,675 probes.
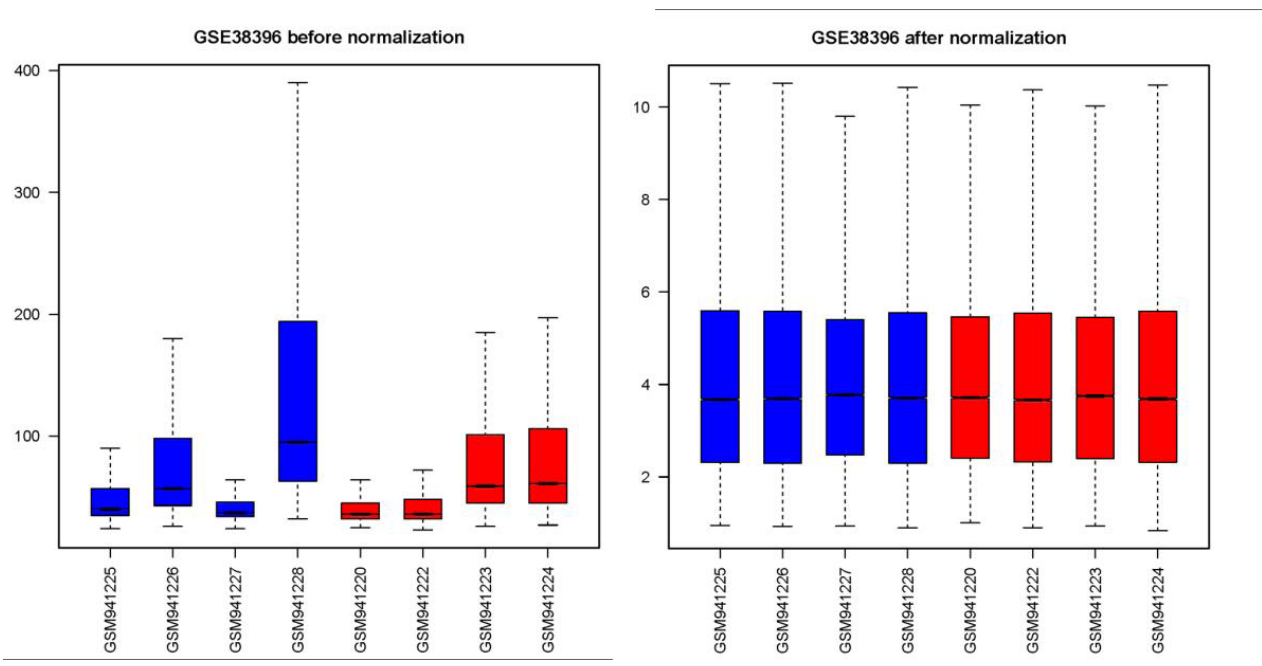

Figure 1. Expression cassette of samples before and after the data preprocessing. The left figure is the cassette before normalization and the right one is the cassette after normalization. The horizontal axis indicates the name of the sample, and the ordinate represents the value of the expression. The black lines in each cassette are the median of data.

\section{Identification and functional annotation of DEGs}

After limma package treatment, a total of 421 DEGs were identified. Among the DEGs, a total of 208 genes were upregulated in skin lymphatic endothelial cells from patients with type II diabetes, while 213 genes were downregulated.

Upregulation of genes can occur in response to trauma, ion balance, and intracellular chemical balance, with biological functions mainly focused on purine nucleotide, lipid, and phospholipid binding, and the metabolic pathways involved lysosomes and complement systems (Table 1). The biological processes involved in downregulation of genes included cell adhesion, regulation of cell proliferation, and metabolism of phosphate, with biological functions mainly focused on GTP, nucleotide, and GTP enzyme 
binding, and the involved metabolic pathways included cancer pathways and hematopoietic cell lineages (Table 2).

Table 1. Gene ontology terms and significant pathways of upregulated genes.

\begin{tabular}{llcc}
\hline Category & Term & Count & P value \\
\hline GOTERM_BP_FAT & GO:0009611 response to wounding & 14 & 0.002305 \\
GOTERM_BP_FAT & GO:0050801 ion homeostasis & 10 & 0.020484 \\
GOTERM_BP_FAT & GO:0055082 cellular chemical homeostasis & 9 & 0.03529 \\
GOTERM_BP_FAT & GO:0006873 cellular ion homeostasis & 9 & 0.032576 \\
GOTERM_BP_FAT & GO:0055080 cation homeostasis & 7 & 0.02372 \\
GOTERM_CC_FAT & GO:0005773 vacuole & 4 & 0.03606 \\
GOTERM_CC_FAT & GO:0031091 platelet alpha granule & 3 & 0.017005 \\
GOTERM_CC_FAT & GO:0031594 neuromuscular junction & 27 & 0.018998 \\
GOTERM_MF_FAT & GO:0017076 purine nucleotide binding & 23 & 0.038385 \\
GOTERM_MF_FAT & GO:0030554 adenyl nucleotide binding & 23 & 0.042868 \\
GOTERM_MF_FAT & GO:0001883 purine nucleoside binding & 11 & 7 \\
GOTERM_MF_FAT & GO:0008289 lipid binding & 5 \\
GOTERM_MF_FAT & GO:0005543 phospholipid binding & 5 & 0.049317 \\
KEGG_PATHWAY & hsa04142:Lysosome & 4 & 0.0070076 \\
KEGG_PATHWAY & hsa04610:Complement and coagulation cascades & 0.036926 \\
\hline
\end{tabular}

Table 2. Gene ontology terms and significant pathways of downregulated genes.

\begin{tabular}{llcl}
\hline Category & Term & Count & P value \\
\hline GOTERM_BP_FAT & GO:0007155 cell adhesion & 21 & 1.21 E-04 \\
GOTERM_BP_FAT & GO:0022610 biological adhesion & 21 & 1.24 E-04 \\
GOTERM_BP_FAT & GO:0042127 regulation of cell proliferation & 20 & 0.001421 \\
GOTERM_BP_FAT & GO:0006793 phosphorus metabolic process & 18 & 0.048379 \\
GOTERM_BP_FAT & GO:0006796 phosphate metabolic process & 18 & 0.048379 \\
GOTERM_CC_FAT & GO:0044459 plasma membrane part & 37 & 0.002536 \\
GOTERM_CC_FAT & GO:0044421 extracellular region part & 23 & 3.18 E-04 \\
GOTERM_CC_FAT & GO:0005615 extracellular space & 14 & 0.024689 \\
GOTERM_CC_FAT & GO:0031982 vesicle & 13 & 0.044238 \\
GOTERM_CC_FAT & GO:0031012 extracellular matrix & 13 & 8.90 E-04 \\
GOTERM_MF_FAT & GO:0042802 identical protein binding & 9 & 0.036255 \\
GOTERM_MF_FAT & GO:0005525 GTP binding & 9 & 0.041886 \\
GOTERM_MF_FAT & GO:0019001 guanyl nucleotide binding & 0.04765 \\
GOTERM_MF_FAT & GO:0032561 guanyl ribonucleotide binding & 8 & 0.04765 \\
GOTERM_MF_FAT & GO:0003924 GTPase activity & 9 & 0.00677 \\
KEGG_PATHWAY & hsa05200:Pathways in cancer & 6.025853 \\
KEGG_PATHWAY & hsa04640:Hematopoietic cell lineage & 0.002388 \\
\hline
\end{tabular}

\section{Relationship between miRNA and DEGs}

A total of 24 miRNAs were extracted from the miRecords database, and a total of 34 target genes were involved. As shown in Figure 2, hsa-miR-124 (PMID: 24247359), hsa-miR-1 (PMID: 24394957), and has-miR-373 (PMID: 21704010) regulated 9, 6, and 5 of the target genes, respectively, and the gene cell-surface glycoprotein 44 (CD44) (PMID: 22499789) was regulated by 4 miRNAs.

These results indicate that the biological process involved in these genes included cell migration, regulation of cell proliferation, cell death, and cell adhesion regulation, among others (Table 3 ). 


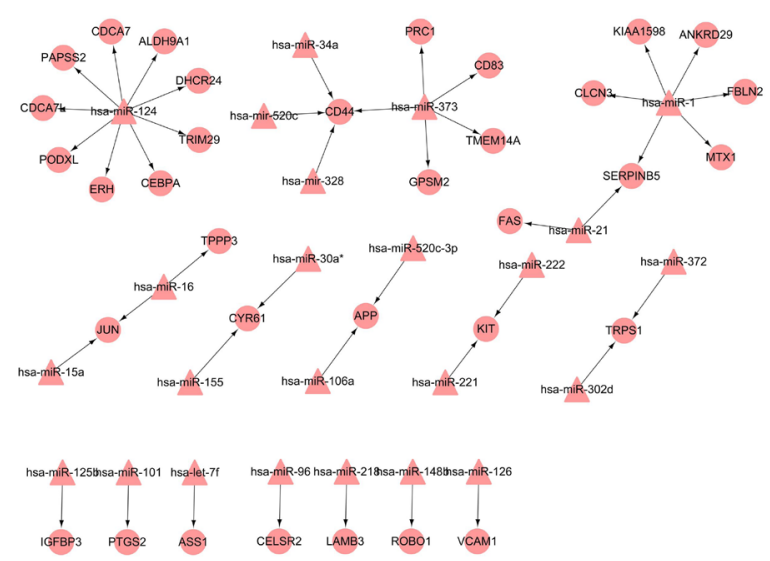

Figure 2. Network between miRNAs and their target genes. The triangles represent miRNAs, the circles represent target genes, and the arrow lines represent regulation relationships.

Table 3. Biological processes involved in target genes affected by miRNA directly.

\begin{tabular}{llcc}
\hline Category & Term & Count & P value \\
\hline GOTERM_BP_FAT & GO:0006928 cell motion & 8 & 6.35 E-05 \\
GOTERM_BP_FAT & GO:0042127 regulation of cell proliferation & 8 & 0.001376 \\
GOTERM_BP_FAT & GO:0043067 regulation of programmed cell death & 8 & 0.001649 \\
GOTERM_BP_FAT & GO:0010941 regulation of cell death & 8 & 0.001685 \\
GOTERM_BP_FAT & GO:0007155 cell adhesion & 7 & 0.00384 \\
\hline
\end{tabular}

\section{Construction and analysis of protein-protein interaction network}

As shown in Figure 3, the protein-protein interaction networks were constituted by 135 nodes and 166 edges.

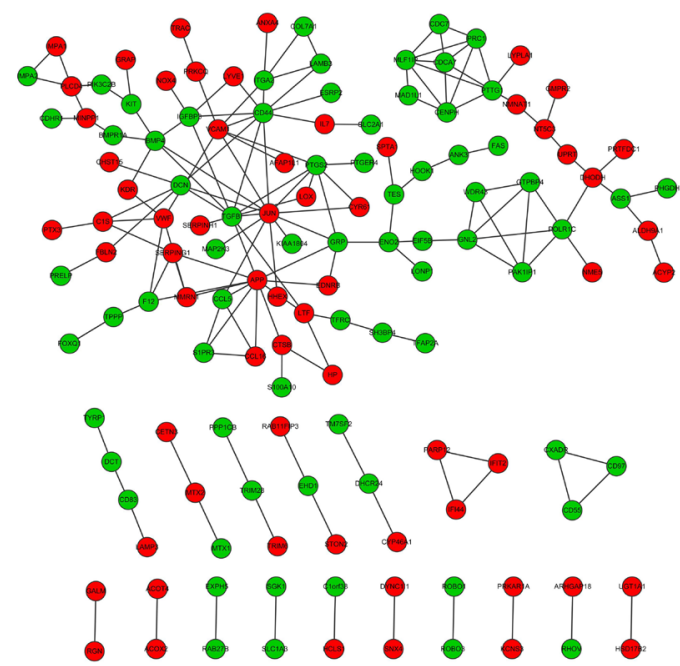

Figure 3. Protein-protein interaction network corresponding to differentially expressed genes. The red circles represent the upregulated proteins and the green circles represent the downregulated proteins. 
After cluster analysis for the protein-protein interaction network, a module was selected $($ density $=0.133$, quality $=0.741, \mathrm{P}$ value $=1.541 \mathrm{E}-6)$. As shown in Figure 4, the module was composed of 25 nodes and 40 edges.

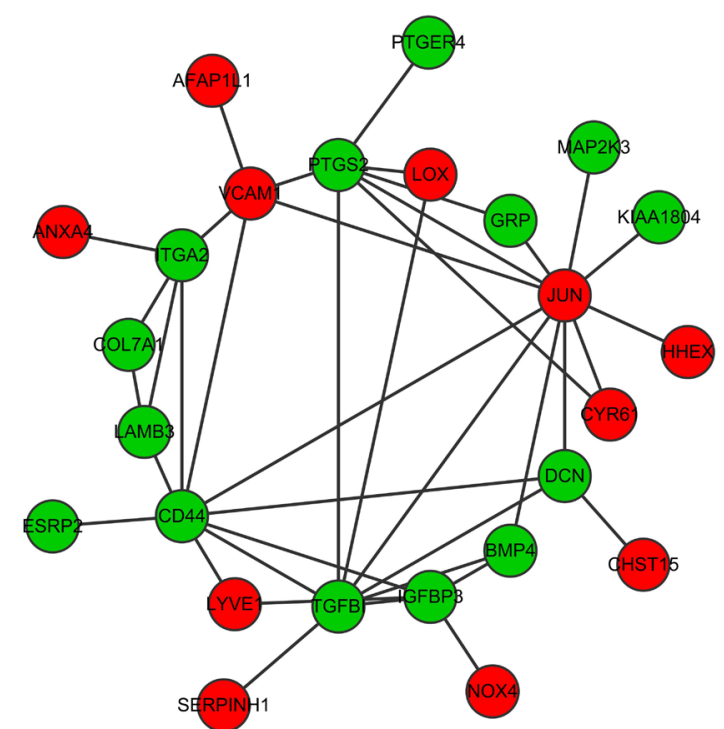

Figure 4. Module in protein-protein interaction network. The red circles represent the upregulated proteins and the green circles represent the downregulated proteins.

DAVID online tools were applied to perform INTERPRO protein domain enrichment analysis for 25 genes in this module (Table 4). The protein domain of the clustering module was mainly concentrated in the insulin-like growth factor-binding protein. Two proteins involved in enrichment were insulin-like growth factor-binding protein 3 (IGFBP3) (PMID: 22884472) and cysteine-rich angiogenic inducer 61 (CYR61) (PMID: 22160564).

Table 4. Protein enrichment of the genes in module network.

\begin{tabular}{llll}
\hline Category & Term & P value & Genes \\
\hline INTERPRO & IPR017891:Insulin-like growth factor binding protein, N-terminal & 0.017157 & IGFBP3, CYR61 \\
INTERPRO & IPR000538:Link & 0.019989 & LYVE1, CD44 \\
INTERPRO & IPR000867:Insulin-like growth factor-binding protein, IGFBP & 0.028438 & IGFBP3, CYR61 \\
\hline
\end{tabular}

\section{DISCUSSION}

Type II diabetes mellitus is characterized by insulin resistance, which may be combined with relatively reduced insulin secretion, and has become 1 of the 3 major diseases affecting human health (Khot and Dhongade, 2014). Diabetes can be accompanied by numerous complications, and skin wound healing in diabetic patients is a major challenge to clinicians (Brownlee, 2005). In our study, a total of 421 DEGs were identified in the skin lymphatic endothelial cells of patients with type II diabetes. The biological processes involved in these upregulated genes (208) included response to trauma, ion balance, and intracellular chemical 
balance, while the biological processes involved in downregulated genes (213) included cell adhesion, regulation of cell proliferation, and phosphate metabolism. Twenty-four miRNAs and 34 target genes were extracted from the miRecords database, and the biological processes related to these genes included cell migration, regulation of cell proliferation, cell death, and cell adhesion regulation, among others.

The hsa-miR-124 miRNA is a small non-coding RNA molecule that has been identified in epithelial cells. In our study, hsa-miR-124 regulated 9 target genes, in which cell division cycle associated 7 and CCAAT/responsive binding protein were related to cell proliferation. Visvanathan et al. (2007) showed that hsa-miR-124 targeted the mRNA of the protein cell division cycle-associated protein 7 , which can enhance transcription factor function in epithelial cells. Makeyev et al. (2007) showed that hsa-miR-124 interacted directly with $C E B P A$ to accelerate cell differentiation foreskin wound healing. The interaction between hsamiR-1 and CD44 was found to be related to skin wound healing in type II diabetes patients. Huang et al. (2008b) found that human miR-373 stimulated cancer cell migration and invasion in vitro and in vivo, and the migration phenotype of miR-373 can be explained by suppression of CD44. CD44 can interact with matrix metalloproteinases (MMPs), which are involved in cell-cell interactions, cell adhesion, and migration (Yu et al., 2002). The expression of MMPs in refractory wounds in diabetes patients was upregulated, resulting in an imbalance of MMPs/ tissue inhibitors of MMPs. miR-1 plays key roles in the development and differentiation of smooth and skeletal muscles, which may induce the expression of apoptotic factors (Yang et al., 2007). The detailed molecular mechanism for serpin peptidase inhibitor, clade B (ovalalbumin), member 5 (SERPINB5) function has been examined in cell proliferation. In the late stage of healing, this hsa-miR-1-related gene in epithelial cells is involved in extracellular matrix (ECM) degradation, which is an important process required for cell invasion and migration (Cervigne et al., 2009). These results indicate that the interactions between miRNAs and target genes are focused on cell migration, regulation of cell proliferation, cell death, and cell adhesion regulation, among others.

After protein-protein interaction network clustering analysis, a module including 25 genes was identified, and INTERPRO protein domain enrichment analysis showed that this clustering module was mainly concentrated in IGFBP3 and CYR61. IGFBP3 is a member of the IGFBP family (Renehan et al., 2004). IGFBP3 protein level was shown to increase during skin wound healing in type II diabetes patients (Saygun et al., 2008). In our study, IGFBP3 was enriched in the protein-protein interaction network. Thus, we speculated that by combining with insulin-like growth factor-1, IGFBP3 can inhibit the activity of this protein to inhibit cell proliferation and further regulate cell mitosis, inhibiting the anti-apoptotic effect of IGF-1. Finally, the apoptotic cells on the wound surface showed increased difficulty in wound healing.

CYR61 is a secreted, ECM-associated signaling protein of the CCN intercellular signaling protein family (Brigstock, 2002). In skin wound healing, CYR61 is highly expressed in the granulation tissue, which may promote the synthesis of the ECM to maintain tissue integrity; however, excessive ECM deposition or degradation can cause abnormal wound healing (Grzeszkiewicz et al., 2002). In our study, CYR61was enriched in the protein-protein interaction network. The abnormal expression of CYR61 in skin wound healing of diabetic patients may induce an imbalance in the ECM, which is an important mechanism for delayed wound healing in diabetes.

In conclusion, our results show that CD44 and SERPINB5 are the latest miRNA traget genes related to delayed wound healing in diabetes, providing a potential link between these 
molecules and skin wound healing in type II diabetes. The proteins IGFBP3 and CYR61 are important in skin wound healing in patients with diabetes, providing a basis for developing methods that can be used to treat skin refractory wounds of type II diabetes.

\section{ACKNOWLEDGMENTS}

Research supported by the Foundation of Shanghai 9th People's Hospital (\#2012A11). We thank Fenghe (Shanghai) Information Technology Co., Ltd., for assistance with the study.

\section{REFERENCES}

Brem H and Tomic-Canic M (2007). Cellular and molecular basis of wound healing in diabetes. J. Clin. Invest. 117: 1219-1222.

Brigstock DR (2002). Regulation of angiogenesis and endothelial cell function by connective tissue growth factor (CTGF) and cysteine-rich 61 (CYR61). Angiogenesis 5: 153-165.

Brownlee M (2001). Biochemistry and molecular cell biology of diabetic complications. Nature 414: 813-820.

Brownlee M (2005). The pathobiology of diabetic complications a unifying mechanism. Diabetes 54: 1615-1625.

Buerger C, Richter B, Woth K, Salgo R, et al. (2012). Interleukin-1 $\beta$ interferes with epidermal homeostasis through induction of insulin resistance: implications for psoriasis pathogenesis. J. Invest. Dermatol. 132: 2206-2214.

Cervigne NK, Reis PP, Machado J, Sadikovic B, et al. (2009). Identification of a microRNA signature associated with progression of leukoplakia to oral carcinoma. Hum. Mol. Genet. 18: 4818-4829.

Chan P-K, Zhao M, Che C-T and Mak E (2008). Cytotoxic acylated triterpene saponins from the husks of Xanthoceras sorbifolia. J. Nat. Prod. 71: 1247-1250.

Franceschini A, Szklarczyk D, Frankild S, Kuhn M, et al. (2013). STRING v9. 1: protein-protein interaction networks, with increased coverage and integration. Nucleic Acids Res. 41: D808-D815.

Goto F, Goto K, Weindel K and Folkman J (1993). Synergistic effects of vascular endothelial growth factor and basic fibroblast growth factor on the proliferation and cord formation of bovine capillary endothelial cells within collagen gels. Lab. Invest. 69: 508-517.

Grzeszkiewicz TM, Lindner V, Chen N, Lam SC-T, et al. (2002). The angiogenic factor cysteine-rich 61 (CYR61, CCN1) supports vascular smooth muscle cell adhesion and stimulates chemotaxis through integrin $\alpha 6 \beta 1$ and cell surface heparan sulfate proteoglycans. Endocrinology 143: 1441-1450.

Haemmerle M, Keller T, Egger G, Schachner H, et al. (2013). Enhanced lymph vessel density, remodeling, and inflammation is reflected by gene expression signatures in dermal lymphatic endothelial cells in type 2 diabetes. Diabetes 62: 2509-2529.

Huang da W, Sherman BT and Lempicki RA (2008a). Systematic and integrative analysis of large gene lists using DAVID bioinformatics resources. Nat. Protoc. 4: 44-57.

Huang Q, Gumireddy K, Schrier M, Le Sage C, et al. (2008b). The microRNAs miR-373 and miR-520c promote tumour invasion and metastasis. Nat. Cell Biol. 10: 202-210.

Irizarry RA, Hobbs B, Collin F, Beazer-Barclay YD, et al. (2003). Exploration, normalization, and summaries of high density oligonucleotide array probe level data. Biostatistics 4: 249-264.

Khot SS and Dhongade SR (2014). Microwave assisted multicomponent synthesis of excellent antidiabetic (type 2) active thiazolidinone derivatives. Proceedings of the National Conference on Drug Designing and Discovery DDD2013. Devchand College, Arjunnagar, India, 77-80.

Liu ZJ and Velazquez OC (2008). Hyperoxia, endothelial progenitor cell mobilization, and diabetic wound healing. Antioxid. Redox. Sign. 10: 1869-1882.

Lu KV and Bergers G (2013). Mechanisms of evasive resistance to anti-VEGF therapy in glioblastoma. CNS Oncol. 2: 49-65.

Makeyev EV, Zhang J, Carrasco MA and Maniatis T (2007). The microRNA miR-124 promotes neuronal differentiation by triggering brain-specific alternative pre-mRNA splicing. Mol. Cell 27: 435-448.

Rapraeger AC, Krufka A and Olwin BB (1991). Requirement of heparan sulfate for bFGF-mediated fibroblast growth and myoblast differentiation. Science 252: 1705-1708.

Renehan AG, Zwahlen M, Minder C, O'Dwyer ST, et al. (2004). Insulin-like growth factor (IGF)-I, IGF binding protein-3, and cancer risk: systematic review and meta-regression analysis. Lancet 363: 1346-1353.

Sakai S and Tagami H (2010). Dry skin in diabetes mellitus and in experimental models of diabetes. In: Textbook of Aging Skin. Springer, New York, pp. 653-661. 
Sanges R, Cordero F and Calogero RA (2007). oneChannelGUI: a graphical interface to Bioconductor tools, designed for life scientists who are not familiar with R language. Bioinformatics 23: 3406-3408.

Santarpia L, Lippman SM and El-Naggar AK (2012). Targeting the MAPK-RAS-RAF signaling pathway in cancer therapy. Expert Opin. Ther. Targets 16: 103-119.

Saygun I, Karacay S, Serdar M, Ural AU, et al. (2008). Effects of laser irradiation on the release of basic fibroblast growth factor (bFGF), insulin like growth factor-1 (IGF-1), and receptor of IGF-1 (IGFBP3) from gingival fibroblasts. Laser Med. Sci. 23: 211-215.

Shannon P, Markiel A, Ozier O, Baliga NS, et al. (2003). Cytoscape: a software environment for integrated models of biomolecular interaction networks. Genome Res. 13: 2498-2504.

Spravchikov N, Sizyakov G, Gartsbein M, Accili D, et al. (2001). Glucose effects on skin keratinocytes implications for diabetes skin complications. Diabetes 50: 1627-1635.

Visvanathan J, Lee S, Lee B, Lee JW, et al. (2007). The microRNA miR-124 antagonizes the anti-neural REST/SCP1 pathway during embryonic CNS development. Genes Dev. 21: 744-749.

Wang Y, Krivtsov AV, Sinha AU, North TE, et al. (2010). The Wnt/ $\beta$-catenin pathway is required for the development of leukemia stem cells in AML. Science 327: 1650-1653.

Xiao F, Zuo Z, Cai G, Kang S, et al. (2009). miRecords: an integrated resource for microRNA-target interactions. Nucleic Acids Res. 37: D105-D110.

Yang B, Lin H, Xiao J, Lu Y, et al. (2007). The muscle-specific microRNA miR-1 regulates cardiac arrhythmogenic potential by targeting GJA1 and KCNJ2. Nat. Med. 13: 486-491.

Yu WH, Woessner JF, Mcneish JD and Stamenkovic I (2002). CD44 anchors the assembly of matrilysin/MMP-7 with heparin-binding epidermal growth factor precursor and ErbB4 and regulates female reproductive organ remodeling. Genes Dev. 16: 307-323. 